skin not responding well to standard treatment.Genetic tests helps in making a diagnosis in the child and detection of carrier state in mother who is asymptomatic. This helps in counselling regarding the peripartum risks associated with future pregnancies and the recurrence risk for the offsprings.

\section{EPIDEMIOLOGY OF RESPIRATORY VIRAL INFECTION ADMISSION AMONG CHILDREN WITH DOWN SYNDROME WITHIN THE FIRST 2 YEARS OF LIFE}

Kavitha Sothirasan, Kee Thai Yeo, Amudha Jayanthi Anand. Singapore

\subsection{6/bmjpo-2021-RCPCH.242}

Background Respiratory viral infections (RVI) among children with Down syndrome (DS) is a common cause of hospital admissions. Due to the underlying co-morbidities, the severity and length of hospitalization is high in this population.

Objectives We estimated and described the burden of RVI admission among infants with Down syndrome within the first 2 year of life.

Methods This is a single-centre cohort study that includes all infants diagnosed with Down Syndrome in our centre. Data on laboratory-confirmed RVI were extracted from the microbiology database and linked to the hospital Down syndrome database. Clinical data and details of hospital admission from 2006-2017 were extracted.

Results There was a total of 34 RVI admissions from 29/227 children $(12.8 \%)$ in this cohort during the study period. Seven children (24.1\%) had more than 1 RVI. Respiratory Syncytial Virus (RSV) was the main causative pathogen, accounting for $61.8 \%$ of all admissions (21/34). Parainfluenza virus (6/ $34,17.6 \%)$ and Metapneumovirus (5/34, 14.7\%) infection were the other common infections. Five infants (14.7\%) required admission to the high dependency unit/intensive care unit, all with RSV infection. Thirteen infants (38.2\%) required respiratory support during their admission, ranging from nasal cannula to continuous positive airway pressure (CPAP). The median length of hospitalization was 6 days (range 2-41 days) and the median length of stay in the high dependency/intensive care unit was 8 days (range 3-18 days). A total of 29/34 $(85 \%)$ who had co-morbidities, of which 6 had more than 1 morbidity.

Conclusions Up to $13 \%$ of children with Down Syndrome required admission due to RVI in our cohort. RSV infections was the predominant causative infection, accounting for up to $62 \%$ of all admission and all admission to high-dependency/ intensive care unit.

\section{REDUCING DELAYS IN THE TIME TO FIRST-FEED FOR BABIES WITH POLYHYDRAMNIOS: A QUALITY IMPROVEMENT PROJECT AT A UK DISTRICT GENERAL HOSPITAL}

Timothy Shields, Olatejumoye Knee, Antonia Katana, Igor Fierens, Catherine Taylor. UK

10.1136/bmjpo-2021-RCPCH.243
Background Polyhydramnios is an obstetric complication with increasing incidence due to increased prevalence of maternal risk factors such as diabetes. Nasogastric-tube (NGT) placement has been used to confirm oesophageal patency and exclude associated anomalies such as oesophageal atresia. Delayed time-to-first-feed has considerable negative impacts with delayed bonding and increased risk of neonatal hypoglycaemia.

Objectives To assess the cause of delays to first-feed for babies born with polyhydramnios, compare our practice with that of other neonatal units in the UK and implement changes to reduce time to first-feed.

Methods We sought to identify a practice consensus in the postnatal assessment of babies born with polyhydramnios using a convenience sample of UK hospital local guidelines.

We then retrospectively evaluated the time intervals to firstfeed for all patients born with polyhydramnios over a threemonth period at our hospital. The primary outcome measure was time-to-first-feed from birth with a target of one hour as is commonly accepted in neonatal practice.

Data was anonymised at collection. The PDSA methodology was used to implement sequential changes to practice and repeated measures to improve performance. The project was registered locally.

Results Our convenience sample included 18 UK Neonatal units, of which 13 were Level 3 intensive care units and the remainder were local neonatal or special-care baby units. Only 2 units (11\%) did NGT placement with $\mathrm{x}$-ray for all babies born with polyhydramnios. Over half $(56 \%, n=10 / 18)$ of all units carried out NGT placement but only performed confirmation $\mathrm{x}$-ray if high-risk features were present or if acceptable $\mathrm{pH}$ aspirate unobtainable. The remaining third $(33 \%, n=6 / 18)$ performed no investigations for the sole purpose of postnatal evaluation of polyhydramnios if no highrisk features.

Between February-April 202035 babies were born with polyhydramnios who all underwent NGT placement on NICU and $\mathrm{x}$-ray. The mean minimum time-to-first-feed was 2.76 hours.

Initial interventions were then implemented with junior neonatal doctors trained in NGT placement on NICU and $\mathrm{x}$ ray only done if acidic $\mathrm{pH}$ unobtainable. Re-audit data of 13 patients in July-August 2020 after the first cycle found a reduction in mean time-to-first-feed of one hour to 1.78 hours.

Subsequently implemented changes include: adopting a newly designed local protocol in-line with best available evidence including junior doctors placing NGT on labour ward to avoid separation; educating maternity and medical staff on the changes; ongoing practical skills teaching; and engaging in multidisciplinary discussion to improve patient care collectively.

Further data from August 2020-January 2021 showed that although overall mean time to first feed was essentially static at 1.83 hours, for those only needing an NGT it had dropped to 1.26 hours from 1.83 hours in prior cycle.

Since the change to only doing $\mathrm{x}$-ray if needed, less than half of patients $(n=14 / 31)$ of combined patients from past two PDSA cycles required $\mathrm{x}$-ray confirmation resulting in overall reduced radiation exposure and cost. We will now target reducing time to first-feed for those babies requiring an $\mathrm{x}$ ray. 
Conclusions Sequential interventions involving staff education and an evidence-based protocol have significantly improved outcomes for patients with polyhydramnios.

\section{A PATIENT WITH AUTISTIC SPECTRUM DISORDER AND 17P12 DUPLICATION - LITERATURE REVIEW}

Sophie Hurrell, Hani Ayyash. UK

10.1136/bmjpo-2021-RCPCH.244

Background There is an increased awareness in the role of microarray analysis in conditions such as ADHD and autism due to arising association of genetic mutations with these diagnoses as risk factors. We have a patient who has been recently diagnosed with autistic spectrum disorder with duplications in the short arm of chromosome 17 with breakpoints within $17 \mathrm{p} 12$.

Objectives The purpose is to use the medical literature to search for other cases of $17 \mathrm{p} 12$ duplication to see if there is any phenotypic resemblance between the genetic mutation and neuropsychiatric disorders.

Methods We conducted a literature search for other additional cases of $17 \mathrm{p}$ duplication as well as specifically looking for 17 p12 duplication. We used database engines such as PubMed, National Centre for Biotechnology Information and the Cochrane Library as well as using information from Unique.

Results $17 \mathrm{p} 12$ duplication is a rare disorder with approximately 50 people having been diagnosed in the medical literature. With $17 p$ there are four groups that patients tend to fall into depending on specific breakpoints. With $17 \mathrm{p} 12$ can have an association with Charcot-Marie Tooth Type 1a disease if the peripheral myelin protein (PMP22) gene is also included. Duplications with less than $1 \mathrm{Mb}$ are strongly associated with autistic spectrum disorder.

Due to small numbers there is very little data and literature regarding $17 \mathrm{p}$ duplication in general and even less on $17 \mathrm{p} 12$ specifically. Each patient has a unique phenotypic presentation with varying signs and symptoms. This includes behavioural difficulties (such as autistic features and hyperactivity), intellectual disability and unique facial features.

Our patient initially presented with features of learning difficulties, social communication difficulties, sleep disturbances, repetitive behaviours and being investigated for possible dyspraxia. The neonatal period was uneventful and had normal gross motor development. The duplication was approximately $1.3 \mathrm{Mb}$ and was stated on the results form as being associated with Charcot-Marie-Tooth Syndrome Type 1a (CMT1A) (CMIM \#118220). However, it did not specify as to whether the PMP22 gene was included. CMT1A is a $1.5 \mathrm{Mb}$ duplication on $17 \mathrm{p} 12$ and our patient's duplication was measured at $1.3 \mathrm{Mb}$. This was thought to be a co-incidental finding for the referral reason of autistic spectrum disorder. There is no family history of Charcot-Marie-Tooth and our patient has no symptoms of peripheral neuropathy.

Conclusions $17 \mathrm{p} 12$ duplication is a rare genetic mutation with very little cases within the medical literature. However, as chromosomal microarray has become increasingly requested for patients with unexplained intellectual disability or autistic spectrum disorders there may well be further individuals diagnosed in the future.

\section{STREPTOCOCCUS GALLOLYTICUS SUBSPECIES PASTEURIANUS INFECTION IN INFANTS - A SYSTEMATIC REVIEW AND META-ANALYSIS}

Sandra Jaya-Bodestyne, Yee Yin Tan, Rehena Sultana, Kee Thai Yeo, Juin Yee Kong. Singapore

\subsection{6/bmjpo-2021-RCPCH.245}

Background Streptococcus bovis is a commensal colonic pathogen that is found in both human and animals. Streptococcus bovis biotype $\mathrm{II} / 2$, which is currently known as streptococcus gallolyticus subspecies (ssp) pasteurianus (SGP), is associated with sepsis and meningitis in neonates and infants. While this is uncommon, it has been associated with long term morbidity and mortality.

Objectives The aim of this study is to describe the clinical course and outcomes of infants with SGP infection.

Methods This is a systematic review and meta-analysis of studies identified from PubMed/Medline, Embase, Scopus, Web of Science, Google Scholar, and Cochrane Library. The following $\mathrm{MeSH}$ keywords were used: 'streptococcus gallolyticus', 'streptococcus bovis', 'newborn', 'infant'. Studies were included if they described infants with culture-proven streptococcus gallolyticus (in blood or cerebrospinal fluid) up to 1 year of life. Data extracted included perinatal factors, clinical presentation, investigations, treatment received, and outcomes. Subgroup analyses were performed according to early and late onset of infection, and preterm compared to term infants.

Results A total of 42 articles were identified, which included a total of 97 cases, of which 59 had streptococcus bovis, and 38 with SGP infection (i.e. streptococcus bovis biotype II/2). Of the 97 cases, they were predominantly term infants $(65.7 \%)$ male $(54.5 \%)$, and were born via vaginal delivery (67.4\%). The most common clinical symptom at presentation was lethargy $(63.3 \%, 95 \%$ CI $45.1 \%-78.4)$, fever (60.3\%, 48.3-71.2\%)), irritability (57.6\%, 35.1-77.4\%), tachypnea $(58.1 \%, 40.4-73.9 \%)$ and respiratory distress (48.3\%, 21.9-68.0\%). There were 28 early-onset infections ( $£ 3$ days of life), which commonly presented as bacteremia $(64.3 \%)$. Compared to late-onset infection, neonates with early-onset infection presented with more respiratory symptoms [pooled-risk difference for tachypnea 41.7\% (11.2$72.2 \%)$, respiratory distress $32.4 \% \quad(0.5-64.2 \%)]$. The median inflammatory markers at onset of symptoms were not raised - median leukocyte 7000/microL (3490-16100/ microL), neutrophils 45\% (34-60\%), C-reactive protein $9.6 \mathrm{mg} / \mathrm{dL}(5.2-13.48 \mathrm{mg} / \mathrm{dL}))$. CSF studies in neonates with meningitis showed predominant pleocytosis (median leukocyte $\left.824 / \mathrm{mm}^{3}\left(13-3016 / \mathrm{mm}^{3}\right)\right)$. Most cases were susceptible to penicillin $(66.1 \%, 53.6-76.8 \%)$ and were treated with a penicillin derivative with good recovery. The reported cases of meningitis had cranial imaging findings of intraventricular hemorrhage, ventriculitis, hydrocephalus, as well as frontal and subdural effusion. There were 4 mortalities - two presented and died on the first day of life, and two had a late-onset presentation and deteriorated before antibiotics could be given.

Conclusions SGP is an important cause of sepsis and meningitis in neonates. Penicillin derivative is an effective medication for SGP with limited reports of resistance and outcomes appear to be favourable. 